21st Particles and Nuclei International Conference (PANIC 2017)

International Journal of Modern Physics: Conference Series

Vol. 46 (2018) 1860076 (6 pages)

(C) The Author(s)

DOI: $10.1142 / \mathrm{S} 2010194518600765$

\title{
The LUCID-2 Luminometer
}

\author{
V. Hedberg (for the ATLAS-LUCID collaboration) \\ Physics Department, University of Lund, Sweden \\ Vincent.Hedberg@cern.ch
}

Published 3 May 2018

\begin{abstract}
The LUCID-2 detector is the main online and offline luminosity monitor of the ATLAS experiment. It provides 104 different luminosity measurements from different algorithms for each of the thousands of LHC bunches. The new detector is using the quartz windows of $10 \mathrm{~mm}$ diameter photomultipliers and optical quartz fibers as the Cherenkov medium. A main challenge for a luminometer is to keep the efficiency constant during years of data-taking. LUCID-2 is using an innovative calibration system based on radioactive Bi-207 sources deposited on the quartz window of the readout photomultipliers.
\end{abstract}

\section{Introduction}

The luminosity of the LHC determines the interaction rate at the collision points and is governed by the number of circulating protons and the size of the beam-beam overlap area at the interaction point. If one knows the luminosity, the measurement of the rate for a given process translates directly into the cross section for that process, which is the quantity of interest for comparing with theory and other measurements. An accurate estimation of the luminosity is particularly important for any physics analysis in which a cross section is measured and it is hence of importance to a wide range of different physics analysis in ATLAS. Measurements of the absolute cross section of $W$ and $Z$ bosons have so far been a major motivation for precise luminosity measurements at the LHC since the theoretical errors are particularly small. The interest has now turned to studies of top-quarks where the luminosity error has dominated.

\section{The new LUCID-2 detector}

The LUCID detector ${ }^{1}$ was entirely re-designed in preparation for LHC Run 2 with both a new detector and upgraded electronics. This was necessary in order to cope with the challenging conditions at the LHC center of mass energy of $13 \mathrm{TeV}$ with

This is an Open Access article published by World Scientific Publishing Company. It is distributed under the terms of the Creative Commons Attribution 4.0 (CC-BY) License. Further distribution of this work is permitted, provided the original work is properly cited. 


\section{Hedberg}

only $25 \mathrm{~ns}$ bunch-spacing and up to 80 simultaneous pp-collisions. The new LUCID2 detector $^{2}$ is now the main online and offline luminosity provider in the ATLAS experiment. It provides 104 different luminosity measurements from different algorithms for each of the 2544 LHC bunches that has been colliding so far.

While LUCID-1 used gas as a Cherenkov medium, the new LUCID-2 detector is in a unique way using the quartz windows of $10 \mathrm{~mm}$ diameter Hamamatsu R760 photomultipliers as the Cherenkov medium. The high particle density close to the beampipe where LUCID is located, means that it is a challenge not to have particles hitting the detector in every bunch crossing. Specially constructed photomultipliers with a smaller acceptance have therefore been produced in cooperation with the company. These photomultipliers have a ring of Aluminium deposited between the window and the photocathode. The photomultipliers have been extensively tested with neutrons from reactors, high-intensity radioactive gamma sources, cosmic rays and by running them with large LED signals at a high rate for a long time.

Figure 1 depicts the new LUCID-2 detector. It is a compact design with $16+16$ small $10 \mathrm{~mm}$ diameter R760 photomultipliers in 4 groups around the beampipe and $4+4$ bundles of quartz fibers read out by photomultipliers situated some 1.5 $\mathrm{m}$ away from the detector in a shielded location. The 40 photomultipliers in the two detectors are divided up into 5 separate subdetectors, each consisting of $4+4$ photomultipliers. The main detector is a detector monitored by radioactive bismuth

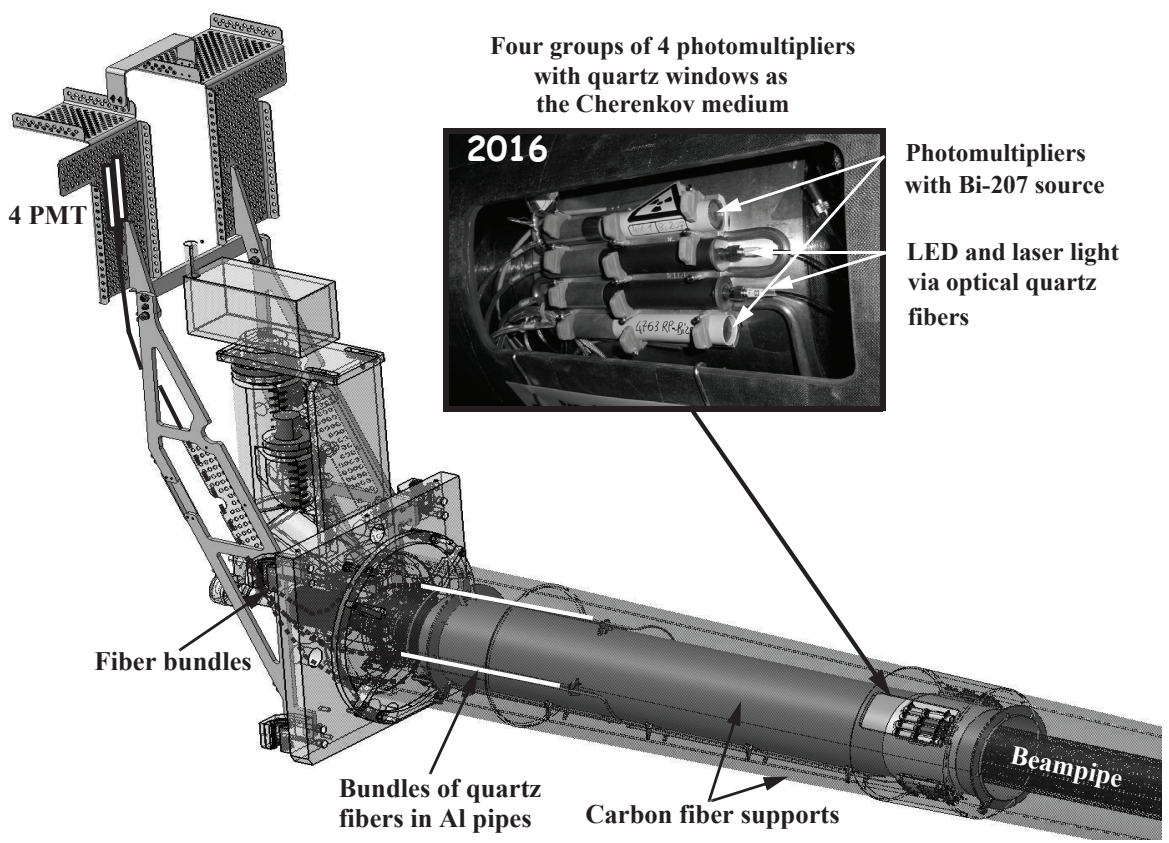

Fig. 1. A schematic view of one of the two LUCID-2 detectors. 16 photomultipliers are grouped 4 by 4 around the beampipe and 4 bundles of quartz fibers go to 4 photomultipliers situated in a shielded location. 

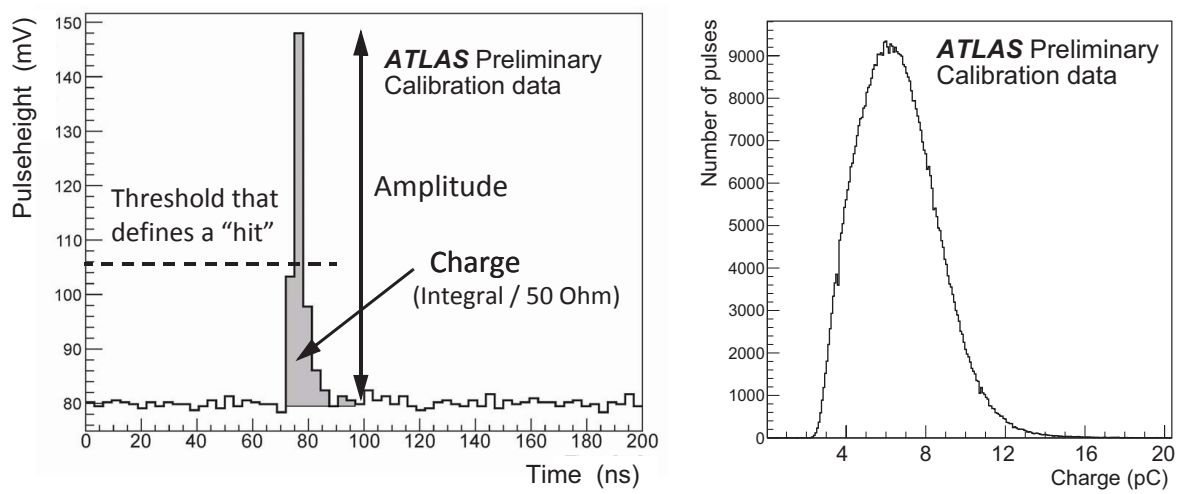

Fig. 2. Left: A typical single pulse from a photomultiplier after it has been digitized by the LUCID electronics. Right: The distribution of pulse charge in a Bi-207 calibration run.

sources. Then there is the detector with modified photomultipliers mentioned earlier. One detector was monitored with LED signals and had as a main purpose to be used in special calibration runs with very low interaction rate where it was feared that the background from radioactive bismuth could be a problem. One detector has been used as a back-up detector that provides independent luminosity measurements for comparison and another has been kept turned off as a spare. Finally there is the fiber detector mentioned earlier.

New purpose-built electronics have been made for LUCID-2 and placed close to the detector in the ATLAS cavern so that the pulses do not become wider than $25 \mathrm{~ns}$ before they are digitized by the electronics. The pulses are measured by flash ADCs that digitize the pulseheight every 3.125 ns. The new electronics do not only count the number of pulses that are above a threshold (so-called hits) but also the pulse amplitude and the integral of the pulses. The measurement of the pulse integral is called a charge measurement since the value of the integral can be regarded as the

$\underline{\text { LED Diffusers }}$

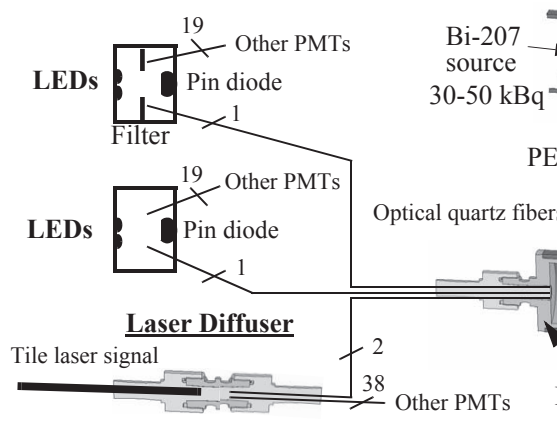

Source monitored photomultiplier

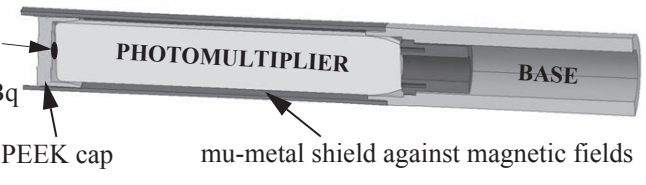

LED monitored photomultiplier

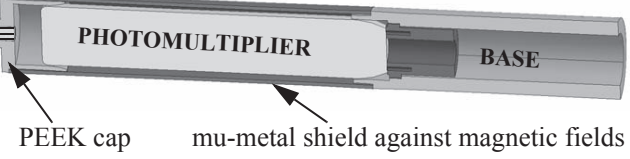

Fig. 3. A schematic illustration of the LUCID calibration systems. 
charge of the pulse if it is divided by the impedance of the electronics, which is 50 Ohm. Figure 2 shows a single photomultiplier pulse after it has been measured by the LUCID electronics and illustrates how hits, amplitude and charge are defined. It can be seen from the figure that the pulse is within the $25 \mathrm{~ns}$ window needed to measure signals from individual LHC bunches.

\section{Monitoring of the detector stability}

A main challenge for a luminometer is to keep the efficiency constant during years of data-taking. LUCID-2 has three different systems that can monitor the photomultiplier gains (see Figure 3). Some of the photomultipliers have LED light monitored by PIN diodes that goes to the photomultipliers via optical quartz fibers. Laser light from the calibration system of the ATLAS TILE calorimeter can also be used. However, LUCID-2 is also using an innovative calibration system based on weak radioactive Bi-207 sources deposited on the quartz window of some of the photomultipliers. The Bi-207 sources produces $1 \mathrm{MeV}$ internal conversion electrons that have sufficient energy to produce Cherenkov light in the quartz window of the photomultipliers.

The Bi-207 system has several advantages over the LED and laser systems. It does not have any components such as LEDs, PIN diodes, optical filters or fibers that can age or get radiation damaged. The main advantage, however, is that the sources produces Cherenkov light of a similar wavelength and intensity compared to that of the Cherenkov light from particles produced in the $p p$-collisions.
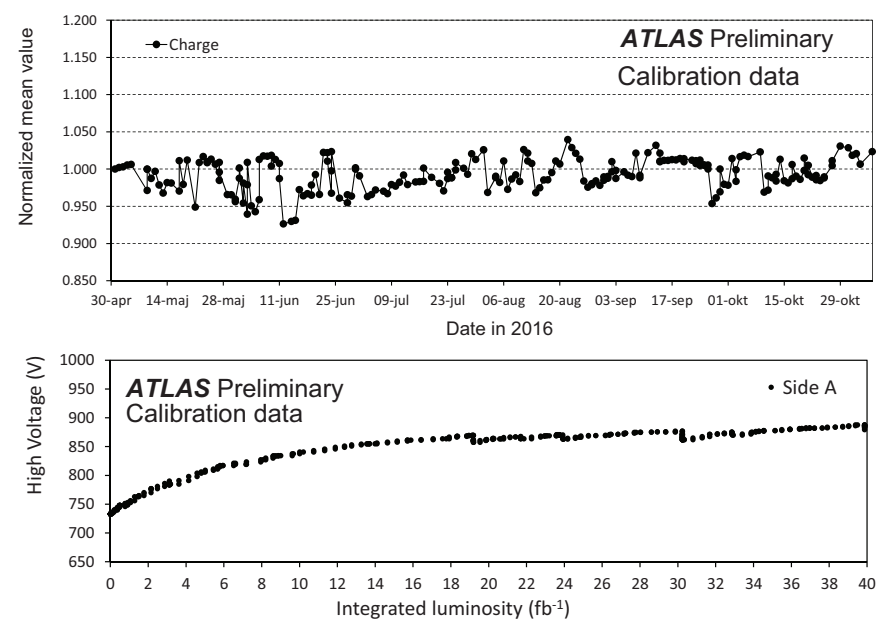

Fig. 4. Top: Measurements with one photomultiplier of the mean charge in Bi-207 calibration runs during 2016. Each point correspond to a measurement in one calibration run. The data is normalized to 1 in the first run. Bottom: The average change of high voltage for 4 photomultipliers during 2016 as a function of the accumulated integrated luminosity. The high voltage was changed automatically as a result of the Bi-207 calibrations. 
Calibration runs with the LED and Bi-207 systems have been carried out between most LHC fills and the mean values of amplitude and charge distributions in these 10-15 minute long runs (see Figure 2) are measured and compared to reference values obtained at the beginning of the year. If there was a significant change of the mean charge, the high voltage to the photomultipliers was automatically changed to keep the photomultiplier gain stable. Figure 4 shows the result of the measurements of the mean charge during 2016. One can see that the mean charge was kept more or less stable during the year. The calibration runs resulted typically in corrections of a couple of volts but over the entire year this added up to a significant increase of the high voltage. This can be seen in the bottom plot in Figure 4 that shows how the high voltage was increased during the year. At the beginning of the year a rapid increase of the high voltage was needed and then later on the photomultipliers stabilized. The same has been observed in 2015 and 2017.

\section{The luminosity analysis}

LUCID is measuring luminosity by sampling the rate of inelastic interactions. At a collider where there is rarely no more than one interaction for every bunch crossing, the luminosity is proportional to the rate of triggers from a detector that is sensitive to inelastic events. The luminosity at the LHC is, however, so high that there are typically many inelastic interactions every time a pair of proton bunches cross each other in ATLAS. The number of interactions occurring in a short time periods follows a Poisson distribution with an average called $\mu$. ATLAS divides the data taking period during a LHC fill up into smaller time-periods called luminosity blocks (LB) that are typically one minute long. The aim is to measure the average luminosity for each colliding bunch pair during these luminosity blocks.

The algorithms used by LUCID to measure the luminosity can be divided up into three main groups: event-counting algorithms, hit-counting algorithms and chargecounting algorithms. In event-counting algorithms the number of bunch crossings in a luminosity block that fulfill certain requirements are counted. In hit-counting the total number of hits is counted while in charge counting the total charge of all pulses in a group of photomultipliers is added up during the LB time-period.

A particular detector and method will in event- and hit-counting mode measure a visible number of interactions $\left(\mu^{\mathrm{vis}}\right)$ that depends on the method's efficiency and acceptance $(\epsilon)$ to detect a single interaction $\left(\mu^{\mathrm{vis}}=\epsilon \mu\right)$. The goal is to measure $\mu^{\mathrm{vis}}$ with as high accuracy as possible during a luminosity block and then calculate the luminosity $\left(L_{\mathrm{LB}}\right)$ from a sum over all colliding bunches:

$$
L_{\mathrm{LB}}=\frac{f_{\mathrm{LHC}}}{\sigma^{\mathrm{vis}}} \sum_{j=1}^{n_{b}} \mu_{j}^{\mathrm{vis}}
$$

where $f_{\mathrm{LHC}}$ is the LHC revolution frequency that is a known quantity. The problem of measuring luminosity can now be factorized into two parts: the absolute calibration in a special LHC fill, i.e., determining $\sigma^{\text {vis }}$, while the second part consists of measuring $\mu^{\text {vis }}$ in all other fills. 
An event-counting algorithm was used as the main algorithm in 2015 but as $\mu$ increased in 2016 it was necessary to switch to a hit-counting algorithm. For both these algorithms a formula has been derived that relates $\mu^{\text {vis }}$ to the measured fraction $(f)$ of events or hits: $\mu^{\text {vis }}=-\ln (1-f)$. In the case of event counting $f=N_{\mathrm{OR}} / N_{\mathrm{BC}}$ is the ratio of the number of bunch crossings for which there is at least one hit somewhere in a group of photomultipliers to that of the total number of bunch crossings and in the case of hit-counting $f=N_{\mathrm{HIT}} / N_{\mathrm{BC}} / N_{\mathrm{PMT}}$, i.e., the measured fraction of hits per bunch crossing and photomultiplier.

The absolute calibration of the luminosity methods, i.e., the determination of $\sigma^{\text {vis }}$, is done in special fills called van der Meer fills in which the beams are being separated in the horizontal and vertical direction while the interaction rate is being measured with a particular algorithm. The basic idea is that the luminosity can be obtained from the width of the two scan curves, the peak $\mu^{\text {vis }}$ values during the scans and the number of protons in the colliding bunches. The latter is measured by a pair of DC current transformers that measure the total LHC current and a pair of fast beam current transformers that measure the fraction of the total current in each bunch.

The statistical errors in a luminosity measurement by LUCID is insignificant and the important question is what systematic error one can achieve in the luminosity measurement. The LUCID luminosity is therefore compared with the luminosity estimated from counting tracks in the inner detector and from the current drawn by three different calorimeter systems in ATLAS. In order to minimize the systematic error, the data from the frequent Bi-207 calibration runs are used to correct the data offline. The track counting measurement is also used to correct the LUCID measurement for pile-up effects and in 2016 also for a $0.7 \%$ drift of the measurement during the beginning of the year. The final offline ATLAS luminosity and the error analysis is therefore using data from five different ATLAS detectors. The total luminosity error estimated in 2015 was $2.1 \%$ and it was $2.2 \%$ in 2016 .

\section{Summary and conclusions}

The LUCID-2 detector is using the quartz windows of photomultipliers and optical quartz fibers as the Cherenkov medium. It is the main online and offline luminosity provider of the ATLAS experiment during LHC run 2. A main requirement of a luminometer is that its detection efficiency is kept constant over long periods of times. The LUCID-2 detection efficiency has been monitored by electrons from radioactive Bi-207 sources that produces Cherenkov light and light from LEDs and lasers. The conclusion is that the source method is providing a more reliable monitoring.

\section{References}

1. ATLAS Collab., 2008 JINST 3, S08003.

2. LUCID Collab. (G.L. Alberghi et al.), 2016 JINST 11, P05014. 